\title{
Microarray analysis of vegetative phase change in maize
}

\author{
Josh Strable ${ }^{1, \dagger}$, Lisa Borsuk ${ }^{2}$, Dan Nettleton ${ }^{3}$, Patrick S. Schnable ${ }^{2}$ and Erin E. Irish ${ }^{1, *}$ \\ ${ }^{1}$ Department of Biological Sciences, University of lowa, lowa City, IA 52242, USA, \\ ${ }^{2}$ Center for Plant Genomics, lowa State University, Ames, IA 50011, USA, and \\ ${ }^{3}$ Department of Statistics, lowa State University, Ames, IA 50011, USA
}

Received 22 July 2008; accepted 25 July 2008; published online 19 September 2008.

*For correspondence (fax +319 335 1069; e-mail erin-irish@uiowa.edu).

${ }^{\dagger}$ Present address: Department of Plant Biology, Cornell University, Ithaca, NY, 14853, USA.

\begin{abstract}
Summary
Vegetative phase change is the developmental transition from the juvenile phase to the adult phase in which a plant becomes competent for sexual reproduction. The gain of ability to flower is often accompanied by changes in patterns of differentiation in newly forming vegetative organs. In maize, juvenile leaves differ from adult leaves in morphology, anatomy and cell wall composition. Whereas the normal sequence of juvenile followed by adult is repeated with every sexual generation, this sequence can be altered in maize by the isolation and culture of the shoot apex from an adult phase plant: an 'adult' meristem so treated reverts to forming juvenile vegetative organs. To begin to unravel the as-yet poorly understood molecular mechanisms underlying phase change in maize, we compared gene expression in two juvenile sample types, leaf 4 and culture-derived leaves 3 or 4 , with an adult sample type (leaf 9) using cDNA microarrays. All samples were leaf primordia at plastochron 6. A gene was scored as 'phase induced' if it was up-or downregulated in both juvenile sample types, compared with the adult sample type, with at least a twofold change in gene expression at a $\boldsymbol{P}$-value of $\leq \mathbf{0 . 0 0 5}$. Some 221 expressed sequence tags (ESTs) were upregulated in juveniles, and 28 ESTs were upregulated in adults. The largest class of juvenile-induced genes was comprised of those involved in photosynthesis, suggesting that maize plants are primed for energy production early in vegetative growth by the developmental induction of photosynthetic genes.
\end{abstract}

Keywords: phase change, leaf development, microarray, maize, plastochron 6, heteroblastic.

\section{Introduction}

Like all multicellular organisms, angiosperms pass through a series of developmental states to complete their life cycles. A particularly prominent transition is that from vegetative growth to flowering, when sexual reproduction occurs (Simpson et al., 1999; Steeves and Sussex, 1988). For this transition to occur, the plant must be an adult. That is, a transition in vegetative growth from juvenile to adult precedes the transition from vegetative to reproductive development (Poethig, 1990). This transition between the juvenile phase and the adult phase is called vegetative phase change (Brink, 1962; referred to as phase change hereafter). In many species, vegetative traits change in coordination with the gain of the ability to flower - adult organs are marked by a pattern of differentiation that is distinct from that of juvenile organs (Hackett, 1985; Kerstetter and Poethig, 1998). Understanding the mechanisms that coordinate the regula- tion of adult vegetative differentiation with the gain of floral competence remains a fundamental challenge in plant biology.

In maize, the shoot meristem initiates a genotype-specified number of leaves prior to producing a terminal inflorescence: the tassel. In most genetic backgrounds, the first four or five leaves are juvenile (Freeling, 1992). Phase change occurs during the differentiation of the next two or three leaves formed, which are referred to as transition leaves. Transition leaves are mosaics of both juvenile and adult tissue (Bongard-Pierce et al., 1996). Leaves initiated after phase change differentiate entirely as adult leaves (Poethig, 1990).

Heteroblastic variation between the juvenile and adult phases in maize is most easily observed through morphological and histochemical differences of the epidermis of a 
fully expanded leaf blade (Bongard-Pierce et al., 1996; Evans et al., 1994; Freeling and Lane, 1994; Moose and Sisco, 1994; Poethig, 1990). The surface of juvenile leaves lacks trichomes (macrohairs), and has a dull blue-green appearance caused by the presence of epicuticular wax. Trichomes are a prominent feature of the surface of adult leaves, which have a glossy appearance because epicuticular wax is substantially reduced, as compared with seedling leaves. Anatomically, juvenile leaves are characterized by weakly crenulated walls of epidermal cells in peridermal view, which appear violet when stained with Toluidine Blue-O. In cross section, epidermal cells of juvenile leaves are round, in contrast to cuboidal in the adult leaves. Epidermal cell walls in adult leaves are characterized by their strong crenulation in peridermal view, and their aquamarine staining with Toluidine Blue-O, the latter indicating higher levels of lignin compared with juvenile leaves. Juvenile and adult leaf blades also differ with respect to other components of the cell wall, with juvenile blades having higher total uronosyls and glucose, but lower levels of neutral sugars, xylose, ferulate and ferulate dimers (Abedon et al., 2006).

Several mutations have been described in maize that hasten or delay the appearance of adult-phase traits. Recessive, loss-of-function mutations in the glossy15 (g/15) gene cause the precocious expression of epidermal traits associated with the adult phase (Evans et al., 1994; Moose and Sisco, 1994). The g/15 gene encodes a protein that contains a domain with significant homology to the DNAbinding domain of APETALA2 (AP2; Moose and Sisco, 1996), which is an Arabidopsis transcription factor. As is the case for AP2 (Aukerman and Sakai, 2003), gl15 is a target of the microRNA miR172 (Lauter et al., 2005). The loss-of-function maize early phase change (epc) mutation dramatically reduces the number of juvenile and transition leaves, thereby causing the precocious expression of adult traits and early flowering (Vega et al., 2002). In contrast, Teopod2 (Tp2) and Corngrass 1 (Cg1) are dominant, gain-of-function mutations that extend the expression of a suite of juvenile traits into the adult phase (Poethig, 1988). The recent cloning of $C g 1$ by Chuck et al. (2007) revealed that it encodes two tandemly arrayed miR156 genes. Thirteen putative SQUAMOSA-PROMOTER-BINDING-PROTEIN-LIKE (SPL) genes in maize, which have targets sites for miR156, have been identified (Chuck et al., 2007). Loss-of-function mutations that decrease levels of bioactive gibberellic acid (GA), such as dwarf1 (d1), also prolong the juvenile phase, and, in addition, delay the onset of the adult phase and flowering (Evans and Poethig, 1995).

Phase change in maize can also be altered experimentally by culturing the shoot apex (Irish and Nelson, 1988). Culturederived plants recapitulate normal shoot development, producing juvenile, transition and adult nodes that are similar in number to, and are qualitatively indistinguishable from, those observed for seed-derived plants. Regardless of whether the shoot apex was derived from a juvenile or an adult plant, the first formed leaves have juvenile characters (Irish and Karlen, 1998; Orkwiszewski and Poethig, 2000): i.e. the morphogenetic program of the shoot is 'reset' upon excision and culture. Thus, the relationship between the age of the meristem and its developmental program are uncoupled through culture-induced rejuvenation (Irish and Karlen, 1998; Irish and McMurray, 2006).

Here, we report the identification of genes differentially expressed in a phase-induced manner in leaf primordia at plastochron 6 (P6) in maize by the microarray analysis of juvenile leaf 4 , adult leaf 9 and juvenile leaves 3 or 4 from culture-rejuvenated plants. We identified 221 and 28 genes that were upregulated in the juvenile and adult phases, respectively. The largest class of juvenile-induced genes was comprised of those involved in photosynthesis. Whereas photosynthetic genes have been long known to be induced by light, this observation suggests that in order to prime maize plants for energy production early in vegetative growth, the induction of photosynthetic genes relies on developmental cues.

\section{Results}

To identify genes that may be responsible for the different patterns of phase-specific differentiation, we compared gene expression profiles of juvenile leaf 4 (L4) and adult leaf 9 (L9) primordia. As a control for the juvenile state, we included in our comparison a third sample, culturerejuvenated leaf 3 or $4(\mathrm{RL} 3 / 4)$ primordia. In this way we could eliminate genes that were up- or downregulated early in seedling development, such as those that might be associated with germination, for example, but that would not be truly associated with the juvenile phase. Six independent biological replicates for $L 4, L 9$ and $R L 3 / 4$ were harvested from primordia at P6. RNA extracted from these samples was reverse transcribed, and each target cDNA population was labeled with either a Cy3 or Cy5 florescent dye, in accordance with the experimental design (see Experimental procedures). Dye-labeled RNA populations from the individual sample replicates were co-hybridized to cDNA microarrays that contained 7886 informative elements. Each hybridized microarray was scanned six times, and each time the laser power and photomultiplier tube gain for each dye channel was increased.

\section{Identification of candidate phase-related genes}

Differentially expressed expressed sequence tags (ESTs) were identified using two criteria: a $P$-value of $\leq 0.005$, and an expression ratio that was greater than or equal to 2.0. Estimated false discovery rates (FDR) of $<2.3 \%$ were calculated for those ESTs with $P$-values $\leq 0.005$ (Figures 1 and S1). 
Points in Figure 1 to the right of the vertical line represent ESTs for which expression differences were highly significant $(P \leq 0.005)$. Points above the upper or below the lower horizontal lines in each plot represent ESTs that show a twofold or greater difference in expression. Hundreds of ESTs showed significant differences in expression in the three pairwise comparisons ( $L 4$ versus $L 9$, L9 versus $R L 3 / 4$ and $R L 3 / 4$ versus $L 4$; Figure 1). Further filtering of the data

(a)

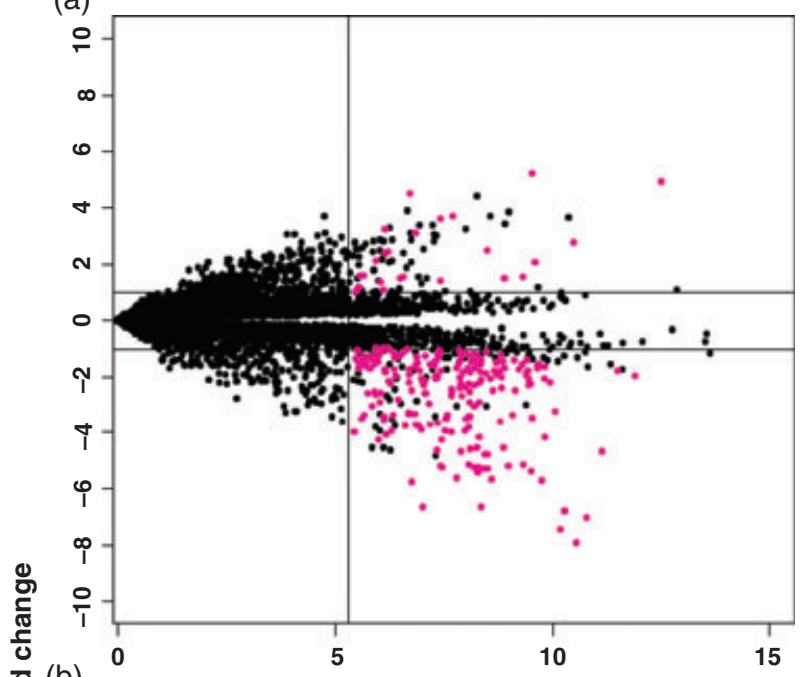

음 (b)

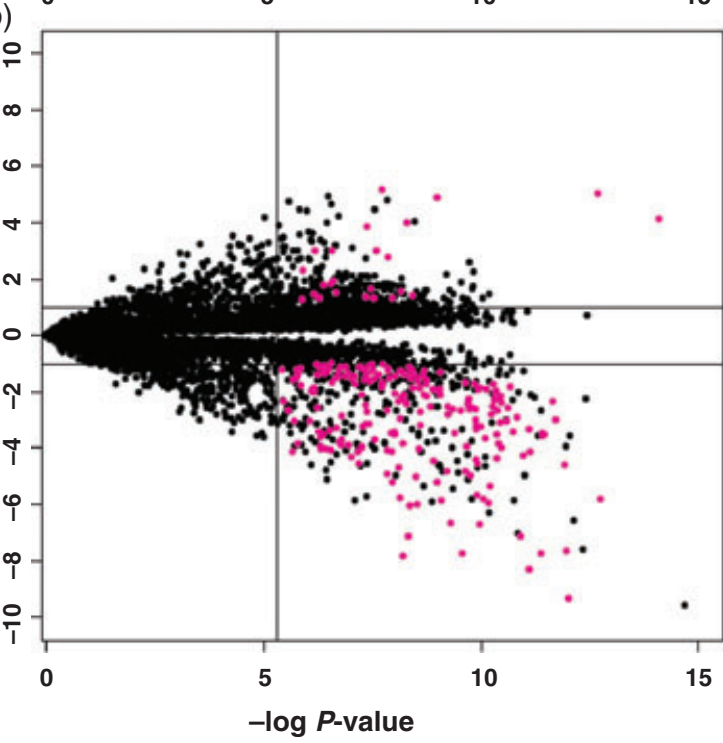

Figure 1 . Expressed sequence tags (ESTs) differentially expressed between juvenile leaf 4 , adult leaf 9 and culture-rejuvenated leaves $3 / 4$ in the lowintensity scan data set.

$(a, b)$ Differences in EST expression levels were plotted against $P$-values derived from a Student's $t$-test. Horizontal lines indicate a difference in gene expression of twofold or more. The vertical line corresponds to a $P$-value of 0.005. Magenta dots in the lower and upper quadrants indicate juvenileinduced and adult-induced ESTs, respectively. The estimated false discovery rates were 2.2 and $0.5 \%$ for the data sets presented in (a) and (b), respectively.

(a) Adult leaf 9 compared with juvenile leaf 4 .

(b) Adult leaf 9 compared with culture-rejuvenated leaves 3 or 4 . sets was accomplished by applying a third criterion: an EST was classified as phase-induced only if it was up- or downregulated in both $L 4$ and $R L 3 / 4$, as compared with $L 9$. These criteria yielded $221 \mathrm{ESTs}$ that were upregulated in juvenile samples, and 28 ESTs that were upregulated in adult samples (Figure 2; Tables 1, 2 and S1).

\section{Expression level comparisons between the seed-derived juvenile state and the culture-rejuvenated state}

Although we defined a gene as being phase-induced if it showed at least a twofold difference in expression between the two phases, no other constraints were applied to the data set: i.e. we did not specify how much greater than twofold the upregulation of the expression should be. To determine the extent to which gene expression mirrors morphological similarity, we compared the expression profiles of L4 versus

(a)

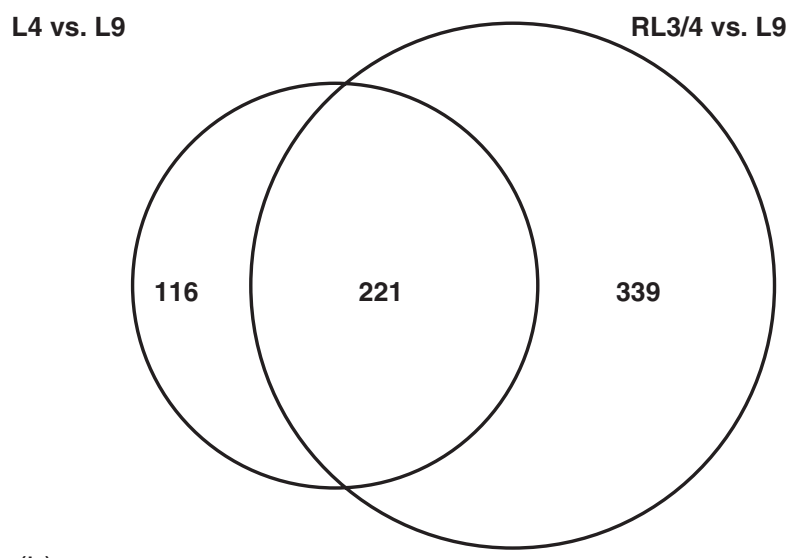

(b)

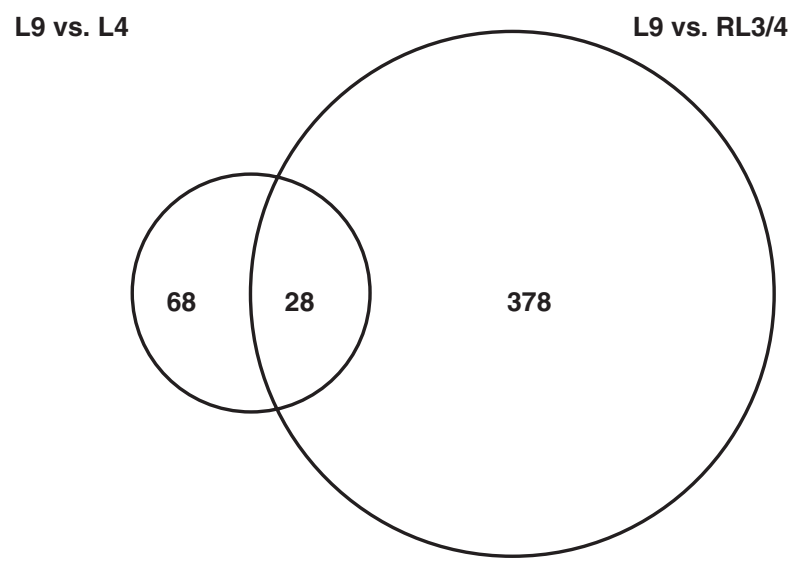

Figure 2. Venn diagrams of juvenile- and adult-induced data sets. (a) Juvenile-induced expressed sequence tags (ESTs; overlap) derived from juvenile leaf 4 (L4) versus adult leaf 9 (L9) and culture-rejuvenated leaves 3 or $4(\mathrm{RL} 3 / 4)$ versus $\mathrm{L} 9$ data sets.

(b) Adult-induced ESTs (overlap) derived from L9 versus $L 4$ and $L 9$ versus RL3/ 4 data sets. 
Table 1 Selected expressed sequence tags (ESTs) that were upregulated in both juvenile leaf 4 and rejuvenated leaves $3 / 4$ compared with adult leaf $9^{a}$

\begin{tabular}{|c|c|c|c|c|c|c|c|c|c|}
\hline \multirow{3}{*}{$\begin{array}{l}\text { EST } \\
\text { GenBank AC }\end{array}$} & \multirow{3}{*}{$\begin{array}{l}\text { Top BLASTX annotation } \\
\text { [GenBank AC (score; } e \text {-value; species)] }^{\mathrm{b}}\end{array}$} & \multicolumn{4}{|c|}{ Low-scan set ${ }^{c}$} & \multicolumn{4}{|c|}{ High-scan set ${ }^{c}$} \\
\hline & & \multicolumn{2}{|c|}{$L 4$ versus $L 9^{d}$} & \multicolumn{2}{|c|}{$R L 3 / 4$ versus $L 9^{d}$} & \multicolumn{2}{|c|}{$L 4$ versus $L 9^{d}$} & \multicolumn{2}{|c|}{$R L 3 / 4$ versus $L 9^{d}$} \\
\hline & & $P$-value $e^{\mathrm{e}}$ & Ratio $^{f}$ & $P$-value $\mathrm{e}^{\mathrm{e}}$ & Ratio $^{f}$ & $P$-value $e^{\mathrm{e}}$ & Ratio $^{f}$ & $P$-value $e^{\mathrm{e}}$ & Ratio $^{f}$ \\
\hline BM339253 & $\begin{array}{l}\text { Immunophilin, putative/FKBP-type pept } \\
\text { idyl-prolyl cis-trans isomerase, putative } \\
\text { [NP_568067.1 (172; 2e-41; } \\
\text { A. thaliana)] }\end{array}$ & $2.06 \mathrm{E}-05$ & 132.00 & $1.83 \mathrm{E}-05$ & 141.11 & $6.44 \mathrm{E}-04$ & 10.46 & $4.65 \mathrm{E}-04$ & 11.63 \\
\hline BM080125 & $\begin{array}{l}\text { Photosystem II subunit PsbS1 } \\
\text { [NP_001105228.1 (73.9; 3e-12; Z. mays)] }\end{array}$ & $2.36 \mathrm{E}-04$ & 100.97 & 7.03E-05 & 214.72 & $8.03 \mathrm{E}-04$ & 13.16 & $3.21 \mathrm{E}-04$ & 18.48 \\
\hline BM074536 & $\begin{array}{l}\text { NADP-malic enzyme [AAP33011.1 } \\
(150 ; 7 \mathrm{e}-35 ; Z \text {. mays })]\end{array}$ & $1.15 \mathrm{E}-03$ & 55.49 & $2.41 \mathrm{E}-04$ & 143.23 & $9.60 \mathrm{E}-04$ & 6.39 & $4.68 \mathrm{E}-05$ & 15.46 \\
\hline BG840776 & $\begin{array}{l}\text { One helix protein [AAM22751.1 (115; } \\
\text { 2e-24; D. antarctica)] }\end{array}$ & $2.52 \mathrm{E}-04$ & 43.62 & 4.71E-05 & 104.48 & $6.79 \mathrm{E}-04$ & 4.04 & $1.79 E-05$ & 9.00 \\
\hline BM267937 & $\begin{array}{l}\text { Photosystem-I reaction center subunit-II, } \\
\text { chloroplast precursor (Photosystem-I 20- } \\
\text { kDa subunit) (PSI-D) [P36213.1 (203; 4e-51; } \\
\text { H. vulgare)] }\end{array}$ & $5.67 \mathrm{E}-04$ & 37.91 & $1.99 \mathrm{E}-04$ & 64.80 & $3.77 \mathrm{E}-04$ & 8.88 & $5.95 \mathrm{E}-05$ & 15.73 \\
\hline DV550281 & RISBZ4 [BAD26199.1 (157; 8e-37; O. sativa)] & $5.94 \mathrm{E}-04$ & 37.56 & $9.04 \mathrm{E}-05$ & 101.79 & - & - & - & - \\
\hline BG840818 & $\begin{array}{l}\text { Putative protodermal factor [BAD26174.1 } \\
(93.2 ; 1 \mathrm{e}-17 ; \text { O. sativa)] }\end{array}$ & $1.26 \mathrm{E}-04$ & 36.75 & $1.26 \mathrm{E}-04$ & 36.76 & $7.40 \mathrm{E}-05$ & 5.49 & $5.65 \mathrm{E}-05$ & 5.81 \\
\hline DV491668 & $\begin{array}{l}\text { Chlorophyll } a / b \text { binding protein } 48 \text {, } \\
\text { chloroplast precursor (LHCIl type-I CAB- } \\
\text { 48) (LHCP) [000827.1 (490; 1e-136; } \\
\text { Z. mays) }]^{9}\end{array}$ & $2.47 \mathrm{E}-04$ & 18.15 & $5.86 \mathrm{E}-05$ & 32.06 & $6.68 \mathrm{E}-04$ & 2.76 & $2.90 \mathrm{E}-05$ & 4.51 \\
\hline DV494632 & $\begin{array}{l}\text { Homeobox protein HD1, putative, } \\
\text { expressed [ABF93721.1 }(114 ; 2 \mathrm{e}-24 ; \\
\text { O. sativa)] }\end{array}$ & $1.85 \mathrm{E}-03$ & 15.49 & 1.63E-03 & 16.33 & $1.74 \mathrm{E}-03$ & 2.47 & $8.25 \mathrm{E}-04$ & 2.74 \\
\hline BM074151 & $\begin{array}{l}\text { ATNSI (NUCLEAR SHUTTLE } \\
\text { INTERACTING); } \quad \text {-acetyltransferase } \\
\text { [NP_973950.1 (88.2; 2e-16; } \text { A. thaliana)] }\end{array}$ & 3.09E-04 & 14.03 & $1.88 \mathrm{E}-04$ & 16.70 & $1.39 \mathrm{E}-03$ & 2.84 & $1.47 \mathrm{E}-03$ & 2.81 \\
\hline BM341648 & $\begin{array}{l}\text { Putative very-long-chain fatty acid } \\
\text { condensing enzyme CUT1 [BAD15940.1 } \\
(175 ; 1 \mathrm{e}-42 ; \text { O. sativa)] }\end{array}$ & $9.98 \mathrm{E}-04$ & 13.77 & $1.90 \mathrm{E}-03$ & 10.89 & 4.57E-04 & 3.41 & $1.40 \mathrm{E}-03$ & 2.85 \\
\hline BM334653 & $\begin{array}{l}\text { Ribulose bisphosphate carboxylase small } \\
\text { subunit1 [NP_001105294.1 (268; 3e-69; } \\
\begin{array}{ll}\text { Z. mays) }]^{g} & \end{array}\end{array}$ & $5.29 \mathrm{E}-04$ & 11.58 & $2.72 \mathrm{E}-04$ & 14.52 & $2.00 \mathrm{E}-04$ & 6.27 & $7.11 \mathrm{E}-05$ & 8.07 \\
\hline BM074011 & $\begin{array}{l}\text { Chlorophyll } a / b \text { binding protein } \\
\text { [AAC15992.1 (444; 5e-135; O. sativa)] }\end{array}$ & $3.32 \mathrm{E}-04$ & 11.38 & $5.11 \mathrm{E}-05$ & 21.62 & $4.21 \mathrm{E}-04$ & 8.04 & $4.40 \mathrm{E}-05$ & 15.94 \\
\hline BM073471 & $\begin{array}{l}\text { Elongation factor family protein } \\
\text { [NP_851035.1 (91.3; 5e-17; } A \text {. thaliana)] }\end{array}$ & $3.02 \mathrm{E}-04$ & 10.97 & $5.93 \mathrm{E}-04$ & 8.92 & $5.73 \mathrm{E}-04$ & 4.05 & $1.28 \mathrm{E}-03$ & 3.49 \\
\hline BM073418 & $\begin{array}{l}\text { NDH-M (SUBUNIT NDH-M OF } \\
\text { NAD(P)H:PLASTOQUINONE DEHYDRO- } \\
\text { GENASE COMPLEX) [NP_001031804.1 } \\
(196 ; 7 \mathrm{e}-49 ; \text { A. thaliana)] }\end{array}$ & $1.09 \mathrm{E}-03$ & 10.22 & $5.24 \mathrm{E}-04$ & 13.11 & $8.49 \mathrm{E}-04$ & 3.94 & $2.65 \mathrm{E}-04$ & 4.98 \\
\hline BM350700 & $\begin{array}{l}\text { F-box family protein, putative, expressed } \\
\text { [ABF93656 }(101 ; 1 \mathrm{e}-19 ; \text { O. sativa) }]^{\mathrm{g}}\end{array}$ & - & - & - & - & $8.90 \mathrm{E}-04$ & 9.87 & $4.82 \mathrm{E}-03$ & 5.87 \\
\hline BM075132 & $\begin{array}{l}\text { Pore-forming toxin-like protein Hfr-2 } \\
\text { [AAW48295.1 (121; 9e-26; T. aestivum) }]^{\mathrm{g}}\end{array}$ & $1.25 \mathrm{E}-03$ & 9.70 & $6.46 \mathrm{E}-05$ & 28.47 & $1.61 \mathrm{E}-03$ & 6.79 & $4.57 \mathrm{E}-05$ & 21.22 \\
\hline BG842033 & $\begin{array}{l}\text { Catalase isozyme } 3 \text { [P18123.2 }(201 ; 2 \mathrm{e}-50 ; \\
\text { Z. mays)] }\end{array}$ & $2.87 \mathrm{E}-03$ & 8.41 & $3.95 \mathrm{E}-04$ & 16.97 & $4.44 \mathrm{E}-03$ & 3.02 & $8.69 E-05$ & 6.75 \\
\hline BG840924 & $\begin{array}{l}\text { ABC transporter family protein [NP_175 } \\
837.2(73.6 ; 6 \mathrm{e}-12 ; A . \text { thaliana })]\end{array}$ & 7.73E-04 & 7.80 & $1.95 \mathrm{E}-03$ & 6.02 & - & - & - & - \\
\hline DV489835 & $\begin{array}{l}\text { Putative photosystem-I reaction center } \\
\text { subunit IV [BAC84088.1 (144; 2e-33; } \\
\text { O. sativa)] }\end{array}$ & $1.59 \mathrm{E}-03$ & 7.10 & $3.61 \mathrm{E}-04$ & 11.13 & $7.25 \mathrm{E}-04$ & 2.61 & $2.15 \mathrm{E}-05$ & 4.43 \\
\hline BG841780 & $\begin{array}{l}\text { Polyamine oxidase [CAC04002.1 (280; } \\
\text { 6e-74; Z. mays)] }\end{array}$ & $3.18 \mathrm{E}-03$ & 6.06 & $6.89 \mathrm{E}-04$ & 9.57 & $2.68 \mathrm{E}-03$ & 5.68 & $5.26 \mathrm{E}-04$ & 9.00 \\
\hline
\end{tabular}




\begin{tabular}{|c|c|c|c|c|c|c|c|c|c|}
\hline \multirow{3}{*}{$\begin{array}{l}\text { EST } \\
\text { GenBank AC }\end{array}$} & \multirow{3}{*}{ 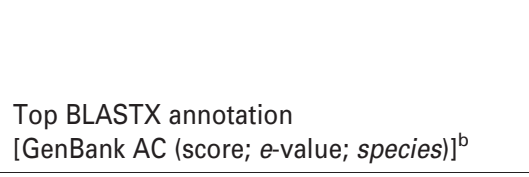 } & \multicolumn{4}{|c|}{ Low-scan set ${ }^{c}$} & \multicolumn{4}{|c|}{ High-scan set ${ }^{c}$} \\
\hline & & \multicolumn{2}{|c|}{ L4 versus $L 9^{d}$} & \multicolumn{2}{|c|}{$\mathrm{RL} 3 / 4$ versus $L 9^{d}$} & \multicolumn{2}{|c|}{$L 4$ versus $L 9^{d}$} & \multicolumn{2}{|c|}{$R L 3 / 4$ versus $L 9^{d}$} \\
\hline & & $P$-value $\mathrm{e}^{\mathrm{e}}$ & Ratio $^{f}$ & $P$-value ${ }^{\mathrm{e}}$ & Ratio $^{f}$ & $P$-value ${ }^{\mathrm{e}}$ & Ratio $^{f}$ & $P$-value ${ }^{\mathrm{e}}$ & Ratio $^{f}$ \\
\hline DV943320 & $\begin{array}{l}\text { Pyruvate, orthophosphate dikinase } \\
\text { [AAA33498.1 }(164 ; 2 \mathrm{e}-39 ; \text { Z. mays })]\end{array}$ & $4.56 \mathrm{E}-04$ & 5.73 & $3.18 \mathrm{E}-05$ & 11.42 & $8.64 \mathrm{E}-04$ & 5.01 & $4.95 \mathrm{E}-05$ & 10.26 \\
\hline BM076073 & $\begin{array}{l}\text { Chalcone synthase C2 (Naringenin- } \\
\text { chalcone synthase C2) [P24825.1 (85.1; } \\
\text { 2e-15; Z. mays)] }\end{array}$ & - & - & - & - & $2.95 \mathrm{E}-03$ & 5.43 & $4.81 \mathrm{E}-03$ & 4.78 \\
\hline DV491239 & $\begin{array}{l}\text { Serine/threonine kinase receptor precursor- } \\
\text { like protein [BAC57307.1 (274; 4e-72; } \\
\text { O. sativa)] }\end{array}$ & - & - & - & - & $4.02 \mathrm{E}-03$ & 5.14 & $4.61 \mathrm{E}-04$ & 9.48 \\
\hline BM073392 & $\begin{array}{l}\text { Arabinoxylan arabinofuranohydrolase } \\
\text { isoenzyme AXAH-II [AAK21880.1 (194; } \\
\text { 4e-48; H. vulgare)] }\end{array}$ & $1.88 \mathrm{E}-04$ & 4.25 & $3.50 \mathrm{E}-05$ & 5.92 & $1.87 \mathrm{E}-03$ & 2.53 & 4.02E-04 & 3.17 \\
\hline DV492715 & $\begin{array}{l}\text { Pentatricopeptide (PPR) repeat-containing } \\
\text { protein [NP_173324.1 (201; 1e-50; } \\
\text { A. thaliana)] }\end{array}$ & $9.20 \mathrm{E}-04$ & 4.23 & $5.21 \mathrm{E}-05$ & 8.09 & - & - & - & - \\
\hline DV491027 & $\begin{array}{l}\text { Putative ABC transporter [AAG52334.1 } \\
(80.1 ; 1 \mathrm{e}-13 ; \text { O. sativa)] }\end{array}$ & $6.60 \mathrm{E}-04$ & 3.97 & $9.83 \mathrm{E}-05$ & 5.84 & - & - & - & - \\
\hline DV491220 & $\begin{array}{l}\text { Restorer of fertility } 2 \text { [NP_001105891.1 (337; } \\
\text { 4e-91; Z. mays)] }\end{array}$ & $2.11 \mathrm{E}-03$ & 3.55 & $7.05 \mathrm{E}-04$ & 4.42 & $1.50 \mathrm{E}-03$ & 2.10 & $8.93 \mathrm{E}-05$ & 2.95 \\
\hline DV492988 & $\begin{array}{l}\text { Putative protein kinase [BAD94332.1 (193; } \\
\text { 6e-48; A. thaliana)] }\end{array}$ & $9.73 \mathrm{E}-04$ & 3.49 & $5.63 \mathrm{E}-05$ & 6.11 & $2.56 \mathrm{E}-03$ & 2.56 & $9.06 \mathrm{E}-05$ & 4.41 \\
\hline DV491620 & $\begin{array}{l}\text { Putative HECT ubiquitin-protein ligase } 3 \\
\text { [BAD07806.1 (90.9; 2e-37; O. sativa) }]^{9}\end{array}$ & $6.84 \mathrm{E}-04$ & 3.06 & $4.43 \mathrm{E}-04$ & 3.28 & $2.21 \mathrm{E}-03$ & 2.24 & $2.55 \mathrm{E}-03$ & 2.20 \\
\hline DV551035 & $\begin{array}{l}\text { Transposon protein, putative, CACTA, } \\
\text { En/Spm sub-class, expressed [ABF97558.1 } \\
(254 ; 3 \text { e-66; O. sativa)] }\end{array}$ & $3.51 \mathrm{E}-03$ & 3.00 & 7.21E-05 & 6.49 & $3.51 \mathrm{E}-03$ & 2.86 & 7.05E-05 & 6.00 \\
\hline DV492584 & $\begin{array}{l}\text { Triose phosphate/phosphate translocator, } \\
\text { chloroplast precursor (cTPT) [P49133.1 } \\
(153 ; 1 \mathrm{e}-35 ; \text { Z. mays)] }\end{array}$ & 7.57E-04 & 2.98 & $1.20 \mathrm{E}-04$ & 4.01 & $1.31 \mathrm{E}-03$ & 2.87 & $1.16 \mathrm{E}-04$ & 4.29 \\
\hline DV942293 & $\begin{array}{l}\text { Auxin response factor } 2 \text { [BAB85913.1 (189; } \\
\text { 2e-46; O. sativa)] }\end{array}$ & $2.91 \mathrm{E}-04$ & 2.76 & $3.26 \mathrm{E}-04$ & 2.72 & $2.58 \mathrm{E}-04$ & 2.42 & $3.21 \mathrm{E}-04$ & 2.36 \\
\hline BM351599 & $\begin{array}{l}\text { Ubiquitin family protein [NP_190104.1 (149; } \\
\text { 7e-34; A. thaliana) }]^{\mathrm{g}}\end{array}$ & $2.83 \mathrm{E}-03$ & 2.56 & $4.39 \mathrm{E}-04$ & 3.43 & - & - & - & - \\
\hline DV490210 & $\begin{array}{l}\text { tpr domain containing protein [ABR25579.1 } \\
(323 ; 9 \mathrm{e}-87 ; \text { O. sativa)] }\end{array}$ & $8.50 \mathrm{E}-04$ & 2.41 & $6.15 \mathrm{E}-04$ & 2.51 & - & - & - & - \\
\hline AW042390 & $\begin{array}{l}\text { Steroid hormone receptor/transcription } \\
\text { factor [NP_187714.1 (94; 4e-18; } \\
\text { A. thaliana)] }\end{array}$ & - & - & - & - & $1.84 \mathrm{E}-03$ & 2.19 & $3.51 \mathrm{E}-03$ & 2.04 \\
\hline
\end{tabular}

${ }^{a}$ ESTs presented in this table are upregulated by at least twofold in both juvenile leaf 4 and rejuvenated leaves $3 / 4$ compared with adult leaf 9 at $P \leq 0.005$

${ }^{\mathrm{b}}$ BLASTX screen of individual EST against the NCBI nr database (February 24, 2008); n.s., no significant BLAST hit (e-value cut-off of 1e-10).

'See Experimental procedures, Data acquisition.

${ }^{d} L 4$, leaf $4 ; R L 3 / 4$, rejuvenated leaves $3 / 4$; $L 9$, leaf 9.

eIndividual $P$-values obtained from the normalized log-scale signal intensity with the mixed linear model.

${ }^{f}$ Antilog of estimated log scale expression differences.

${ }^{9}$ BLASTX screen of individual MAGlv4 (e-value cut-off of e-100) against the NCBI nr database (February 24, 2008).

L9 with those of RL3/4 versus L9. The mean ratio of the $L 4$ versus $\mathrm{L} 9$ comparisons with the $\mathrm{RL} 3 / 4$ versus $\mathrm{L} 9$ was $0.91 \pm 0.03$ ( \pm SEM) (Figure 3a). This suggests that genes that are upregulated in juvenile and culture-rejuvenated samples are expressed at similar levels. A similarly high degree of correlation was calculated for the $L 9$ versus $L 4$ and $L 9$ versus $R L 3 / 4$ comparisons (Figure $3 b$ ), which had a mean ratio of $0.97 \pm 0.06$.

\section{Functional classification of candidate juvenile and adult upregulated ESTs}

The annotation of the juvenile- and adult-induced ESTs was determined by using BLASTX, which compares conceptual translation products with a protein database in GenBank (Tables 1, 2 and S1). Information from the Gene Expression and Visualization Application (GENEVA; http:// 
Table 2 Expressed seqeunce tags (ESTs) that are upregulated in adult leaf 9 compared with both juvenile leaf 4 and rejuvenated leaves $3 / 4^{\mathrm{a}}$

\begin{tabular}{|c|c|c|c|c|c|c|c|c|c|}
\hline \multirow{3}{*}{$\begin{array}{l}\text { EST } \\
\text { GenBank AC }\end{array}$} & \multirow{3}{*}{$\begin{array}{l}\text { Top BLASTX annotation [GenBank AC } \\
\text { (score; } e \text {-value; species)] }\end{array}$} & \multicolumn{4}{|c|}{ Low-scan set ${ }^{c}$} & \multicolumn{4}{|c|}{ High-scan set ${ }^{c}$} \\
\hline & & \multicolumn{2}{|c|}{$L 9$ versus $L 4^{d}$} & \multicolumn{2}{|c|}{$L 9$ versus $R L 3 / 4^{d}$} & \multicolumn{2}{|c|}{$L 9$ versus $L 4^{d}$} & \multicolumn{2}{|c|}{$L 9$ versus $R L 3 / 4^{d}$} \\
\hline & & $P$-value $e^{\mathrm{e}}$ & Ratio $^{f}$ & $P$-value ${ }^{\mathrm{e}}$ & Ratio $^{f}$ & $P$-value ${ }^{\mathrm{e}}$ & Ratio $^{f}$ & $P$-value ${ }^{e}$ & Ratio $^{f}$ \\
\hline DV943135 & $\begin{array}{l}\text { Hypothetical protein OsJ_000126 } \\
{[\text { EAZ10301.1 (101; 6e-21; O. sativa) }]^{\mathrm{g}}}\end{array}$ & $7.19 \mathrm{E}-05$ & 36.80 & $1.26 \mathrm{E}-04$ & 28.95 & $6.89 \mathrm{E}-04$ & 2.89 & $1.46 \mathrm{E}-03$ & 2.60 \\
\hline DV943306 & $\begin{array}{l}\text { 60S ribosomal protein L27 (RPL27C) } \\
\text { [NP_193236.1 (175; 3e-42; A. thaliana] }\end{array}$ & $3.75 \mathrm{E}-06$ & 30.39 & $3.13 \mathrm{E}-06$ & 32.54 & $2.84 \mathrm{E}-03$ & 2.02 & $9.76 \mathrm{E}-04$ & 2.28 \\
\hline Al692021 & $\begin{array}{l}\text { Putative somatic embryogenesis protein } \\
\text { kinase } 1 \text { [BAD68873.1 (119; 1e-25; } \\
\text { O. sativa)] }\end{array}$ & $1.20 \mathrm{E}-03$ & 22.48 & 4.44E-04 & 35.67 & $3.17 \mathrm{E}-03$ & 3.02 & $1.00 \mathrm{E}-03$ & 3.72 \\
\hline DV493121 & $\begin{array}{l}\text { Os05g0356800 [NP_001055294.1 (136; } \\
\text { 2e-29; O. sativa) }]^{9}\end{array}$ & $4.52 \mathrm{E}-04$ & 12.85 & $2.48 \mathrm{E}-04$ & 15.86 & - & - & - & - \\
\hline Al734769 & $\begin{array}{l}\text { Class-III HD-Zip protein } 4 \text {, putative, } \\
\text { expressed [ABF97 } \\
828.1(211 ; 1 \text { 1e-53; O. sativa)] }\end{array}$ & $5.91 \mathrm{E}-04$ & 11.99 & $2.09 \mathrm{E}-03$ & 7.94 & - & - & - & - \\
\hline BM078628 & $\begin{array}{l}\text { Putative growth-regulating factor } 13 \\
{\left[{ }^{2}{ }^{2} \_001106044.1(358 ; 4 \mathrm{e}-96 ; \text { Z. mays) }]^{\mathrm{g}}\right.}\end{array}$ & $2.16 \mathrm{E}-03$ & 9.25 & $6.20 \mathrm{E}-04$ & 14.34 & - & - & - & - \\
\hline BM078110 & $\begin{array}{l}\text { WW domain containing protein, expressed } \\
{[\text { ABA96334.2 (135; 1e-30; O. sativa)] }}\end{array}$ & $1.06 \mathrm{E}-03$ & 8.58 & $1.40 \mathrm{E}-03$ & 7.88 & - & - & - & - \\
\hline DV489742 & $\begin{array}{l}\text { SEC14 cytosolic factor (secretion factor 14) } \\
\text { family protein [CAJ75630.1 (155; 1e-36; } \\
\begin{array}{lll}\text { B. sylvaticum)] } & \end{array}\end{array}$ & $2.82 \mathrm{E}-05$ & 6.79 & $7.61 \mathrm{E}-07$ & 17.63 & - & - & - & - \\
\hline BM072783 & n.s. & - & - & - & - & $4.94 \mathrm{E}-03$ & 5.89 & $3.19 \mathrm{E}-03$ & 6.71 \\
\hline DY576313 & $\begin{array}{l}\text { Protein kinase family protein [NP_973956.1 } \\
(180 ; 1 \mathrm{e}-57 ; \text { A. thaliana) }]^{\mathrm{g}}\end{array}$ & $2.04 \mathrm{E}-04$ & 5.50 & $1.36 \mathrm{E}-03$ & 3.73 & - & - & - & - \\
\hline DV493254 & $\begin{array}{l}\text { Protein kinase family protein [NP_566630.1 } \\
(135 ; 4 \mathrm{e}-29 ; \text { A. thaliana) }]^{\mathrm{g}}\end{array}$ & $1.99 \mathrm{E}-03$ & 5.45 & $5.00 \mathrm{E}-04$ & 7.87 & - & - & - & - \\
\hline DV943127 & $\begin{array}{l}\text { Helix-loop-helix DNA-binding [ABD32380.2 } \\
(78.2 ; 3 \mathrm{e}-12 ; \text { M. truncatula) }]^{\mathrm{g}}\end{array}$ & $2.12 \mathrm{E}-03$ & 5.29 & $2.72 \mathrm{E}-03$ & 4.97 & $1.25 \mathrm{E}-04$ & 2.01 & $1.33 \mathrm{E}-04$ & 2.00 \\
\hline DV943234 & n.s. & $2.62 \mathrm{E}-03$ & 4.30 & $3.88 \mathrm{E}-04$ & 6.79 & $5.83 \mathrm{E}-04$ & 2.09 & $1.45 \mathrm{E}-04$ & 2.43 \\
\hline Al737934 & $\begin{array}{l}\text { Putative lateral organ boundaries (LOB) } \\
\text { domain family }[\text { AAT42184.1 (75.1; 5e-11; } \\
\text { Z. mays) }]^{g}\end{array}$ & $6.86 \mathrm{E}-05$ & 4.22 & $2.76 \mathrm{E}-03$ & 2.39 & - & - & - & - \\
\hline BM333901 & n.s. & - & - & - & - & $1.35 \mathrm{E}-03$ & 3.44 & $3.82 \mathrm{E}-03$ & 2.86 \\
\hline DV493108 & n.s. & $3.50 \mathrm{E}-03$ & 2.99 & $1.45 \mathrm{E}-03$ & 3.50 & $2.48 \mathrm{E}-03$ & 2.91 & 1.39E-03 & 3.20 \\
\hline DV492422 & $\begin{array}{l}\text { Putative growth-regulating factor } 13 \\
{\left[{ }^{2}{ }^{2} \_001106044.1(358 ; 4 \mathrm{e}-96 ; \text { Z. mays) }]^{\mathrm{g}}\right.}\end{array}$ & $3.75 \mathrm{E}-03$ & 2.97 & $1.72 \mathrm{E}-03$ & 3.42 & - & - & - & - \\
\hline DV495600 & $\begin{array}{l}\text { Squamosa promoter-binding-like } \\
\text { protein } 13 \text { [Q6Z461.1 (82; 9e-14; } \\
\text { O. sativa) }]^{9}\end{array}$ & $8.96 \mathrm{E}-05$ & 2.94 & $3.47 \mathrm{E}-04$ & 2.48 & $1.98 \mathrm{E}-04$ & 2.53 & $5.83 \mathrm{E}-04$ & 2.23 \\
\hline DV942770 & n.s. & $1.39 \mathrm{E}-03$ & 2.86 & $2.12 \mathrm{E}-03$ & 2.69 & $6.33 \mathrm{E}-04$ & 2.57 & $1.15 \mathrm{E}-03$ & 2.38 \\
\hline DV492592 & $\begin{array}{l}\text { Hypothetical protein OsJ_017000 } \\
\text { [EAZ33517.1 (208; 2e-52; O. sativa)] }\end{array}$ & $1.38 \mathrm{E}-04$ & 2.83 & $2.20 \mathrm{E}-04$ & 2.67 & - & - & - & - \\
\hline DV942169 & $\begin{array}{l}\text { Hypothetical protein Osl_016629 } \\
{[\text { EAY95396.1 (120; 7e-25; O. sativa) }]^{g}}\end{array}$ & $1.51 \mathrm{E}-03$ & 2.79 & $1.29 \mathrm{E}-03$ & 2.86 & - & - & - & - \\
\hline DV942475 & $\begin{array}{l}\text { Putative FH protein NFH2 [BAB86073.1 } \\
(189 ; 2 \mathrm{e}-46 ; \text { O. sativa)] }\end{array}$ & $5.93 \mathrm{E}-04$ & 2.67 & $2.85 \mathrm{E}-04$ & 2.96 & $4.85 \mathrm{E}-04$ & 2.24 & $1.91 \mathrm{E}-04$ & 2.48 \\
\hline BM075057 & $\begin{array}{l}\text { Cyclin-dependent kinases regulatory } \\
\text { subunit [AAS13367.1 (144; 3e-33; G. max)] }\end{array}$ & $2.35 \mathrm{E}-03$ & 2.52 & $5.68 \mathrm{E}-04$ & 3.12 & - & - & - & - \\
\hline BM073426 & $\begin{array}{l}\text { OSJNBa0041M21.1 [CAD40443.2 (199; } \\
\text { 1e-49; O. sativa)] }\end{array}$ & - & - & - & - & $3.01 \mathrm{E}-03$ & 2.35 & $3.94 \mathrm{E}-03$ & 2.27 \\
\hline DV942221 & $\begin{array}{l}\text { Hypothetical protein OsJ_002940 } \\
{[\text { EAZ13115.1 (85.9; 5e-27; O. sativa) }]^{9}}\end{array}$ & - & - & - & - & $1.82 \mathrm{E}-03$ & 2.29 & $2.38 \mathrm{E}-04$ & 2.99 \\
\hline BG842886 & $\begin{array}{l}\text { LIM transcription factor homolog } \\
\text { [NP001104937.1 (239; 8e-71; Z. mays)] }\end{array}$ & $3.88 \mathrm{E}-03$ & 2.26 & $1.86 \mathrm{E}-03$ & 2.50 & $2.83 E-03$ & 2.18 & $2.20 \mathrm{E}-03$ & 2.25 \\
\hline DV489762 & $\begin{array}{l}\text { Unknown protein [NP_564481.1 }(90.1 ; \\
7 \mathrm{e}-17 ; \text {; } \text {. thaliana)] }\end{array}$ & $2.22 \mathrm{E}-03$ & 2.11 & $5.35 \mathrm{E}-04$ & 2.49 & - & - & - & - \\
\hline BM072828 & $\begin{array}{l}\text { Inorganic pyrophosphatase [NP_001104889.1 } \\
(171 ; 1 \mathrm{e}-56 ; \text { Z. mays })]\end{array}$ & $4.22 \mathrm{E}-03$ & 2.05 & $6.46 \mathrm{E}-04$ & 2.59 & - & - & - & - \\
\hline
\end{tabular}

${ }^{a}$ ESTs presented in this table are upregulated by at least twofold in leaf 9 compared with both leaf 4 and rejuvenated leaves $3 / 4$ at $P \leq 0.005$.

${ }^{\mathrm{b}}$ BLASTX screen of individual EST against the NCBI nr (February 24, 2008); n.s., no significant BLAST hit (e-value cut-off of 1e-10).

${ }^{\circ}$ See Experimental procedures, Data acquisition.

${ }^{d} L 9$, leaf $9 ; L 4$, leaf $4 ; R L 3 / 4$, rejuvenated leaves $3 / 4$

e Individual $P$-values obtained from the normalized log-scale signal intensity with the mixed linear model.

${ }^{\mathrm{f}}$ Antilog of estimated log scale expression differences.

${ }^{9}$ BLASTX screen of individual MAGlv4 (e-value cut-off e-100) against the NCBI nr (February 24, 2008). 

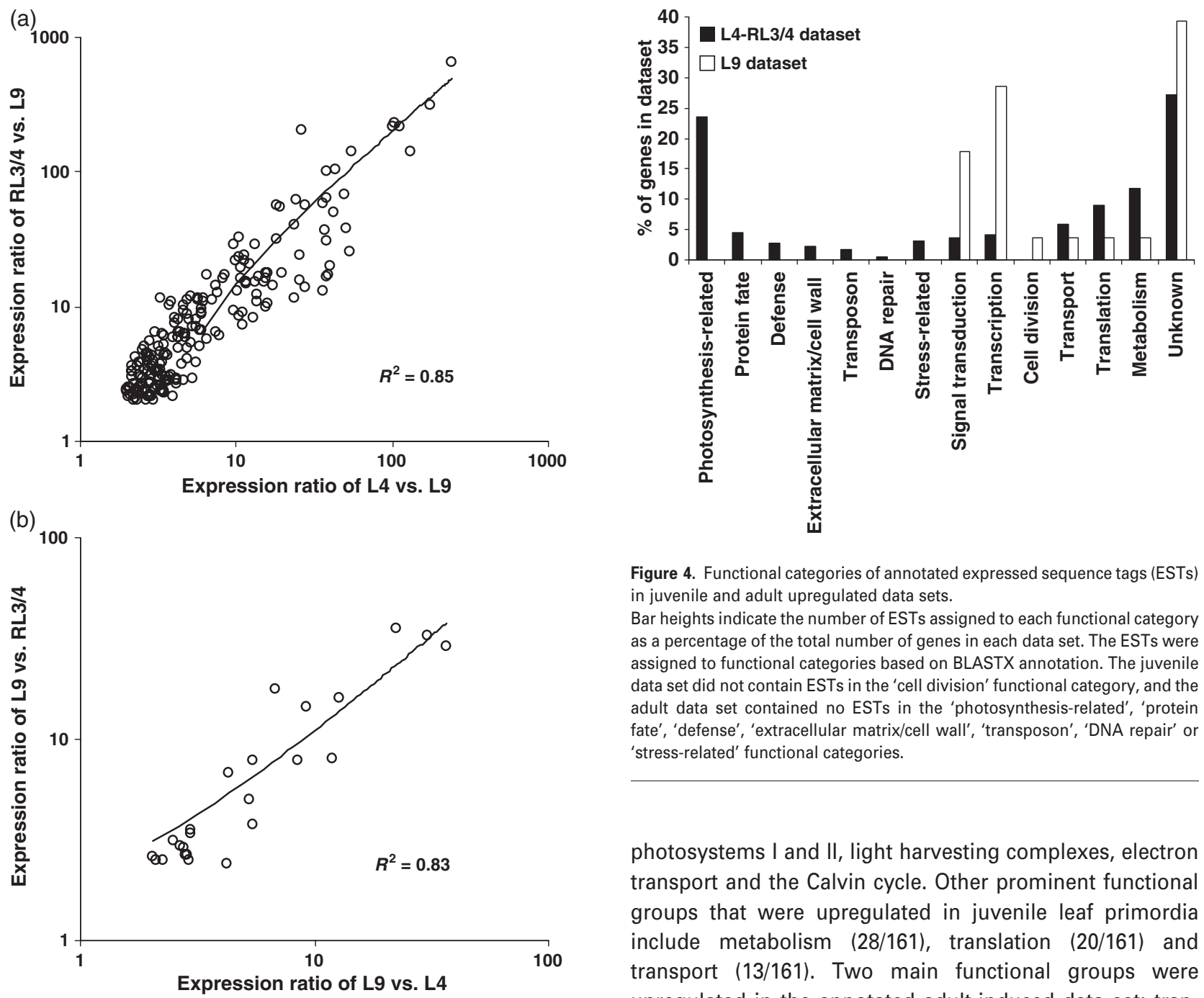

Figure 3. Expression ratios between juvenile leaf 4, adult leaf 9 and culturerejuvenated leaves 3 or 4 comparisons in the low-intensity scan data set are highly correlated.

(a, b) Genes displaying the highest upregulation in each comparison are represented by the upper rightmost points. Note the log scale on both axes. (a) Juvenile upregulated expressed sequence tags (ESTs). The mean ratio of the gene expression values was $0.91 \pm 0.03$ ( $n=206 ; \pm$ SEM).

(b) Adult upregulated ESTs. The mean ratio of the gene expression values was $0.97 \pm 0.06(n=24)$.

sam.truman.edu/geneva/geneva.cgi; Buckner et al., 2007) and The Arabidopsis Information Resource (http:// www.arabidopsis.org) was used to group the ESTs identified in this study into 14 functional categories (Figure 4). Non-annotated genes occupied the largest groups within each data set: $27.1 \%(60 / 221)$ and $39.3 \%(11 / 28)$ for juvenile and adult data sets, respectively. A large fraction of annotated ESTs that were upregulated in juvenile leaves are involved in photosynthesis. This class represents $32.2 \%(52 / 161)$ of the annotated juvenile upregulated ESTs, and includes ESTs that encode putative proteins of

Figure 4. Functional categories of annotated expressed sequence tags (ESTs) in juvenile and adult upregulated data sets.

Bar heights indicate the number of ESTs assigned to each functional category as a percentage of the total number of genes in each data set. The ESTs were assigned to functional categories based on BLASTX annotation. The juvenile data set did not contain ESTs in the 'cell division' functional category, and the adult data set contained no ESTs in the 'photosynthesis-related', 'protein fate', 'defense', 'extracellular matrix/cell wall', 'transposon', 'DNA repair' or 'stress-related' functional categories.

photosystems I and II, light harvesting complexes, electron transport and the Calvin cycle. Other prominent functional groups that were upregulated in juvenile leaf primordia include metabolism (28/161), translation (20/161) and transport (13/161). Two main functional groups were upregulated in the annotated adult-induced data set: transcription (8/17) and signal transduction (5/17). No homologs of juvenile-upregulated photosynthetic genes were found to be upregulated in adult tissues.

\section{Confirmation of microarray data by semiquantitative $R T-P C R$ and RNA blot analyses}

To validate the results of the microarray experiments, the transcript levels of five ESTs upregulated in juvenile leaves, and three ESTs upregulated in adult leaves, were examined by semiquantitative reverse transcriptase (RT)PCR and RNA blot analyses using total RNA prepared from independently isolated, P6-stage leaf primordia samples (Figure 5). Except for some quantitative variation in the relative expression levels, the results were consistent with the phase-induced patterns of EST expression from the microarray data. Two ESTs from each class of phaseinduced ESTs were selected for further analysis, based on their annotation: the juvenile upregulated putative immunophilin/FKBP-type peptidyl-prolyl cis-trans isomerase 


\begin{tabular}{|c|c|c|c|c|c|c|c|c|c|}
\hline \multirow{3}{*}{ BM339253 (30) } & \multicolumn{3}{|c|}{ RT-PCR } & & \multicolumn{3}{|c|}{ RNA blot } & & \\
\hline & Leaf 4 & & Leaf 9 & & Leaf 4 & & Leaf 9 & & \\
\hline & 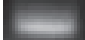 & 0.40 & = & 0.12 & & 0.65 & & 0.03 & \\
\hline BG840776 (27) & $=$ & 0.31 & 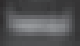 & 0.20 & & 0.18 & & 0.08 & \\
\hline DV491668 (33) & $=$ & 0.33 & & 0.10 & & 0.08 & & 0.01 & Juvenile \\
\hline BM073471 (30) & 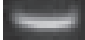 & 0.35 & 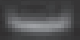 & 0.18 & & 0.11 & & 0.05 & \\
\hline BG840924 (29) & $=$ & 0.71 & 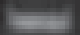 & 0.27 & & 0.08 & & 0.05 & \\
\hline Al692021 (25) & $=$ & 0.39 & $=$ & 0.41 & & 0.32 & & 0.65 & \\
\hline BM078628 (25) & 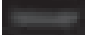 & 0.05 & 0 & 0.10 & & 0.36 & & 0.53 & Adult \\
\hline DV495600 (26) & 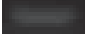 & 0.15 & $x^{2}$ & 0.89 & & 0.02 & & 0.33 & \\
\hline 33) & 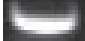 & & 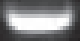 & & & & & & \\
\hline
\end{tabular}

Figure 5. Corroboration of phase-induced differentially expressed genes.

Levels of mRNA isolated from leaf primordia were determined by semi-quantitative RT-PCR and RNA blot analyses. The GenBank accession number appears at the left-hand side. The number of PCR cycles is given in parentheses next to the GenBank accession number. The ratio of the signal relative to the loading control Actin or methylene blue (MB) staining of the 28S rRNA for leaf 4 or leaf 9 is shown to the right-hand side of each RT-PCR or RNA blot image, respectively. Juvenile expressed sequence tags (ESTs) that were upregulated in both leaf 4 (L4) and in culture-rejuvenated leaves 3 or 4 (RL3/4) compared with adult leaf 9 (L9); adult ESTs that were upregulated in L9 compared with both L4 and RL3/4.

(ZmFkb; BM339253) and putative chlorophyll a/b binding protein 48 (Cab48; DV491668), and the adult upregulated putative somatic embryogenesis protein kinase 1 (ZmSek; Al692021) and putative squamosa promoter binding-like protein 13 (ZmSpl; DV495600).

Expression analysis of phase-induced genes in successive leaf primordia from wild-type maize plants

Leaves 4 and 9 were selected as representatives of the juvenile and the adult phases, respectively, for microarray analysis. Whether gene expression in juvenile or adult leaves at other nodes within the two phases of a seedderived plant is accurately represented by the expression profiles generated for leaves 4 or 9 could not be assessed by this profiling strategy. To obtain a more accurate picture of the expression of ZmFkb, Cab48, ZmSek and ZmSpl in the juvenile and adult phases, RNA blot assays were performed on P6-stage primordia of juvenile (1-4), transition (5-7) and adult (8-14) leaves from wild-type plants. In wild-type maize plants, transcripts for the juvenile upregulated $Z m F k b$ were most abundant in juvenile leaf 1 , and decreased precipitously in successive juvenile leaves (Figure 6a,c). ZmFkb expression was not detected in transition (5-7) or adult (814) leaves. Expression of the juvenile upregulated Cab48 was, like $Z m F k b$, strongest in leaf 1, and steadily diminished in subsequent leaves, so that by mid-transition (leaf 6), mRNA levels were uniformly at the threshold of detection by RNA blot analysis (Figure 6b,d). Transcript levels of the adult upregulated $\mathrm{ZmSek}$ were moderately low in juvenile leaves 1-4 and transition leaves 5-7 (Figure 6a,e), but started to increase at leaf 8 , the first adult leaf, accumulated to their highest level in leaf 10 and declined in later adult leaves. The adult upregulated $\mathrm{ZmSpl}$ mRNA was virtually undetectable in juvenile leaves 1-4. ZmSp/ transcript levels increased beginning with transition leaf 5 , and accumulated to high levels in adult leaves 10-14 (Figure 6b,f); in leaves 10 and 11, a second, higher molecular weight transcript was apparent. Thus, whereas some of the microarray identified genes ( $Z m S e k$ and Cab48) simply showed higher expression in leaf primordia of one phase compared with the other, the expression of others (ZmFkb and ZmSpl) could only be detected in one phase.

\section{Discussion}

A number of mutants have been identified that hasten or delay phase change in maize (Chuck et al., 2007; Evans and Poethig, 1995, 1997; Evans et al., 1994; Moose and Sisco, 1994; Poethig, 1988; Vega et al., 2002). Phase change can also be manipulated in maize by use of shoot apex culture, in which the developmental program of an adult meristem is 'reset' back to the juvenile stage when it is excised and cultured (Irish and Karlen, 1998; Irish and Nelson, 1988). That is, the first leaves that an excised meristem initiates differentiate as juvenile leaves, even if the meristem came from an adult plant, such that the shoot recapitulates phase change. Mutant analyses and shoot apex culture experiments have contributed to our understanding of phase change in maize. However, the genes responsible for initiating the transition, 


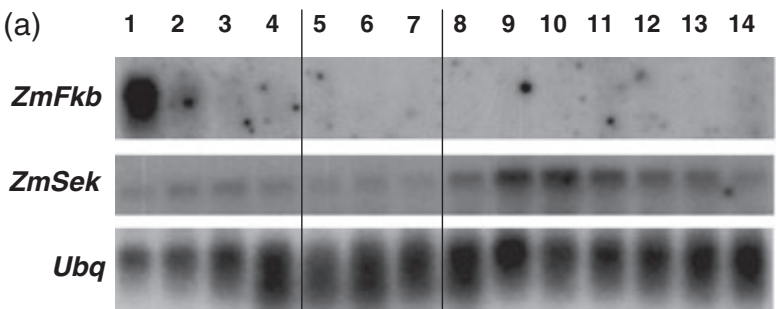

(c)

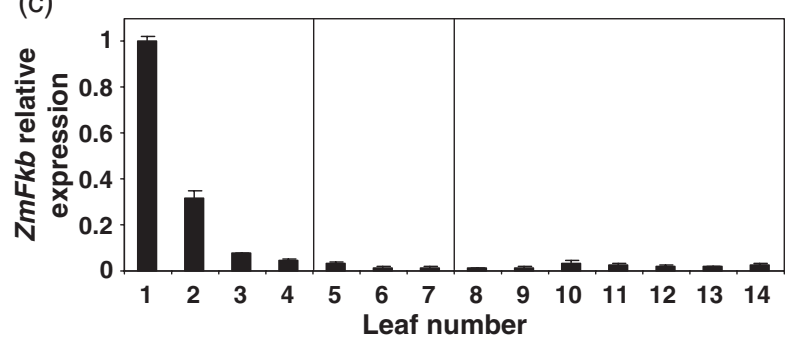

(e)

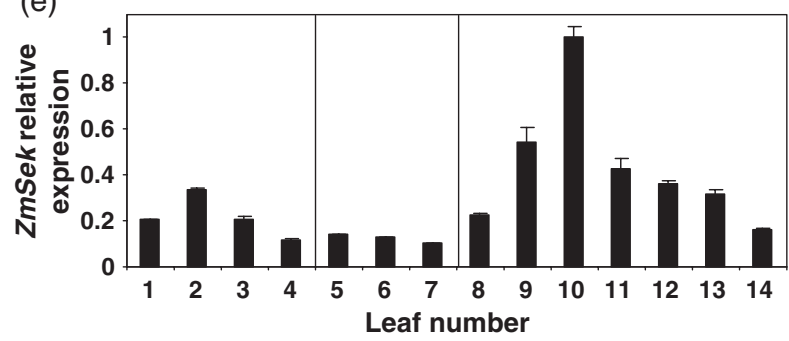

(b)

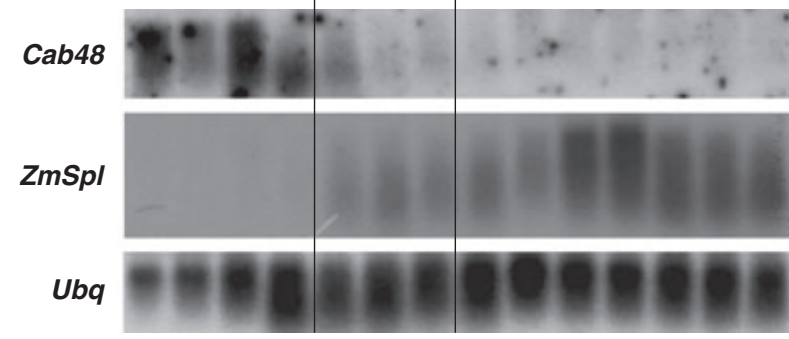

(d)

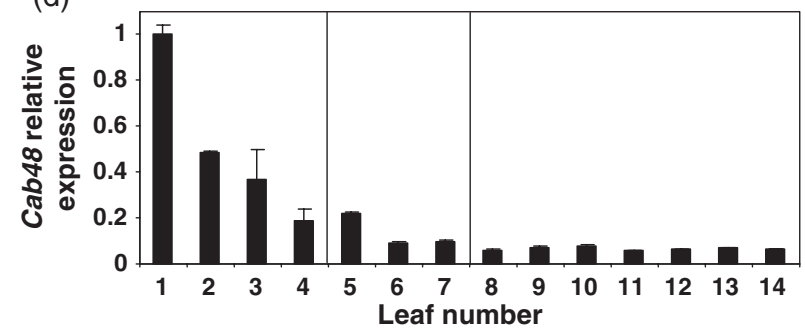

(f)

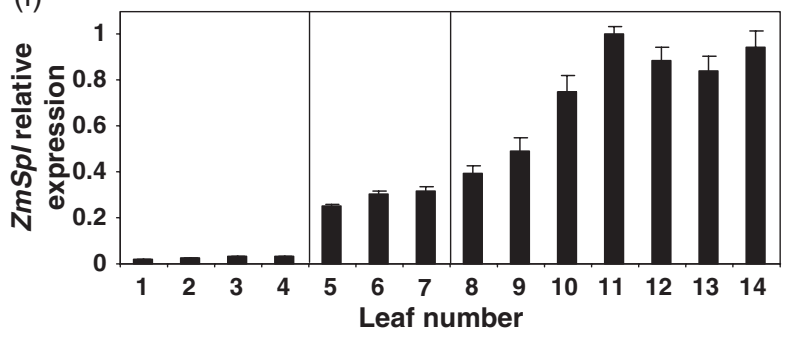

Figure 6. ZmFkb, Cab48, ZmSek and ZmSplexpression in successive leaf primordia of wild-type plants. RNA was isolated from plastochron 6 (P6)-staged primordia from wild-type plants.

The leaf number is indicated above each lane. Vertical lines separate juvenile, transition and adult samples. Total RNA (15 $\mu \mathrm{g}$ ) was loaded per sample. The intensities of the bands in (c-f) were quantified by using NIH IMAGEJ (http://rsbweb.nih.gov/ij), and were normalized to Ubiquitin. The expression levels were quoted relative to the most intense band among individual leaves, arbitrarily set to a value of 1.0. The error bars indicate the standard errors of the mean $(n=2)$. $(a, c) Z m F k b ;(a, e)$ ZmSek; (b, d) Cab48; (b, f) ZmSpl.

as well as those that respond to it, have not yet, for the most part, been identified.

Global level comparisons of gene expression confirm the juvenile phase is recapitulated by shoot apex culture

Juvenile leaves of culture-derived shoots are morphologically and histologically indistinguishable from juvenile leaves of seed-derived plants (Irish and Karlen, 1998; Orkwiszewski and Poethig, 2000). We compared two independent 'juvenile' samples and found that $66 \%$ (221/337) of the ESTs that were upregulated in the $L 4$ versus $L 9$ data set were also upregulated in the RL3/4 versus L9 data set (Figure 2a). In addition, the expression values for the 221 upregulated and the 28 downregulated ESTs showed a strong correlation between the two data sets (Figure 3a,b). The fact that such a large proportion of ESTs from the two data sets are upregulated to similar levels provides support that shoot apex culture 'resets' the genetic networks that regulate juvenile development, and thus validates the experimental approach employed in this study.

\section{Candidate annotated phase-induced ESTs are consistent with the biology of phase-specific differentiation}

Several of the annotated phase-induced ESTs identified in this study complement what is already known about phasespecific differentiation in maize. The blade of juvenile leaves has a dull, blue-green appearance caused by the presence of epicuticular wax on the blade surface. Two juvenile upregulated ESTs are thought to function in epicuticular wax synthesis and epidermis-to-cuticle export of lipids. The putative very long chain fatty acid (VLCFA) condensing enzyme, CUT1 (BM341648), showed a 13.8-fold higher expression in L4 compared with L9 (Table 1). In Arabidopsis, CUT1 is expressed in the epidermis, and is required for the synthesis of VLCFA precursors (Millar et al., 1999). Suppression of CUT1 results in organs with a waxless 
phenotype resembling other mutations, such as those in the eceriferum (cer) loci in Arabidopsis, and in the glossy loci in maize, that cause defects in epicuticular wax biosynthesis or accumulation (Millar et al., 1999). This analysis also revealed a fourfold upregulation of an EST annotated as a putative adenosine triphosphate binding cassette $(A B C)$ transporter (DV491027) in L4 and RL3/4 (Table 1). The CER5 gene in Arabidopsis encodes an $A B C$ transporter required for wax export to the cuticle (Pighin et al., 2004). cer5 mutations result in glossy stems, indicating a reduction in cuticular wax (Pighin et al., 2004).

The juvenile upregulated data set is dominated by genes involved in photosynthesis

Some $30 \%$ of the annotated ESTs in the juvenile data set are involved in photosynthesis (Figure 4). This is true even though P6 primordia are pale yellow, are shaded by a whorl of expanded leaves and are thus unlikely to be undergoing photosynthesis. Langdale et al. (1988) demonstrated that genes involved in photosynthesis are expressed at the early stages of leaf development in maize, independent of light. For example, in an undifferentiated maize leaf blade, RuBPCase large- and small-subunit genes are expressed concurrently with provascular cell divisions, but prior to extensive vascular differentiation. This expression pattern occurs at a stage in leaf development when neither bundle sheath cells nor mesophyll cells have become morphologically distinct, and before chloroplasts can be distinguished by light microscopy (Langdale et al., 1988). The light-independent induction of photosynthesis genes has been documented in Arabidopsis through the expression profiling stages of embryogenesis (Spencer et al., 2007). During the transitions from globular, to heart, to torpedo stages of embryo development, genes involved in energy production comprise the largest functional group that is upregulated, with a strong bias towards the genes encoding components of the photosynthetic apparatus (Spencer et al., 2007).

An EST annotated as a putative Cab48, which showed an 18.2-fold higher expression in L4 than in L9 by microarray analysis, was chosen as a representative of the photosynthesis-related class of genes for examining the pattern of expression in the successive P6-staged leaves of wild-type plants. Developmental regulation of $\mathrm{Cab}$ genes and other photosynthetic genes has been reported in Arabidopsis (Brusslan and Tobin, 1992; Chory et al., 1991), Glycine max (Chang and Walling, 1992), Pisum sativum (He et al., 1994) and Amaranthus hypochondriacus (Ramsperger et al., 1996). Juvenile upregulated expression of Cab has been reported in English ivy (Woo et al., 1994). In wild-type maize plants, Cab48 expression was confined to the primordia of the first five leaves, which, in the genetic background used throughout this study, represents the extent of the juvenile phase (Figure $6 b, d$ ). Whereas light is known to be required for the induction of expression of many genes involved in pigment biosynthesis and in the photosynthetic machinery, developmental cues are also thought to play some role in the regulation of photosynthetic genes. Such developmental regulation could be important for preparing the juvenile seedling for the rapid biogenesis of the photosynthetic apparatus, and thus, energy production, shortly after the emergence of the coleoptile during germination.

\section{ZmFkp expression is regulated in an early juvenile-induced manner}

The expression of a second juvenile upregulated EST, annotated as $Z m F k b$, was examined in successive leaves of wild-type plants. FK506-binding proteins (FKBPs), along with cyclophilins, belong to the immunophilin superfamily (Romano et al., 2005). Members of both the FKBP and cylophilin families function similarly to chaperones in assisting protein folding (Romano et al., 2005). FKBP and cyclophilin proteins have an N-terminal peptidyl prolyl isomerase (PPIase) domain and three tetratricopeptide (TPR) domains located in the $C$ terminus (Pratt et al., 2001; Romano et al., 2005). The PPlase activity isomerizes prolyl bonds from the cis to the trans configuration (Reimer and Fischer, 2002). Mutations in the Arabidopsis cyclophilin 40 ortholog, sqn, eliminate nearly all transition leaves, and dramatically accelerate phase change, indicating a role for the wild-type gene in maintaining the juvenile phase. The $\mathrm{C}$-terminal TPR triplet of SQN is required for interacting with Hsp90 (Berardini et al., 2001). A putative ZmFkb was identified by microarray analysis as being highly upregulated in juvenile samples, with a 132-fold higher expression in L4 compared with L9 (Table 1). RNA blot analysis confirmed its juvenile phase expression, but with the highest levels in L1 and L2 (Figure 6a,c), and much lower levels in all successive leaves, rather than the dramatic difference in expression between $L 4$ and L9, as indicated by the microarray analysis.

\section{$\mathrm{ZmSpl}$ is regulated in a highly phase-induced manner}

The largest classes of annotated adult upregulated ESTs are classified as functioning in signal transduction or transcription. We examined the expression profiles of putative $Z m S e k$ and $Z m S p l$ genes, representing both of these functional categories, in successive P6-staged leaves from wild-type plants. In Arabidopsis, 11 of the 17 SPL genes-including SPL3, SPL4 and SPL5-are targeted by miR156 (Gandikota et al., 2007; Rhoades et al., 2002 Schwab et al., 2005), and in rice, 11 out of the 19 predicted SPL genes-including OsSPL13-have miR156 target sites (Xie et al., 2006). SPL expression in Arabidopsis is antagonized by miR156 in juvenile leaves, where miR156 levels are at their highest (Wu and Poethig, 2006). Upon phase change, miR156 levels decrease and SPL3 mRNA levels 
subsequently increase. In Arabidopsis, SPL3 regulates the expression of a subset of adult traits, such as abaxial trichome distribution and petiole length (Wu and Poethig, 2006). Few members of the SPL family have been characterized in maize. Mutant analyses of the maize SPL genes liguleless1 ( $\lg 1)$ and teosinte glume architecture (tga) indicate that these genes are critical for leaf and glume development, respectively (Moreno et al., 1997; Wang et al., 2005). $\lg 1$ encodes a nuclear-localized protein with a squamosa promoter binding protein (SBP) domain (Moreno et al., 1997), and functions to promote epidermal cell fate with expression in leaf primordia at or prior to P6 (Moreno et al., 1997). Recently, it was found that $\mathrm{Cg} 1^{+}$encodes an miR156 RNA (Chuck et al., 2007). Thirteen potential SPL genes, including tga, that are expected to be targets of $\mathrm{Cg}^{+}$ were identified (Chuck et al., 2007). We found that the level of $\mathrm{ZmSpl}$ mRNA increased in successive leaves, beginning with the early transition L5 in wild-type plants (Figure $6 \mathrm{~b}, \mathrm{f}$ ). $Z m S P L$ is a putative ortholog of OsSPL13, which groups with $S P L 3 / 4 / 5$ of Arabidopsis in an unrooted tree based on the protein sequence of the SBP domain (Xie et al., 2006). The similarity among the expression profiles of $Z m S p l$ in maize (this study) and SPL3/4/5 in Arabidopsis, which are targeted and cleaved by miR156 in a phase-induced manner (Wu and Poethig, 2006), suggests that these are regulated by similar mechanisms, and have conserved functions in establishing the adult phase in these two angiosperm species.

\section{Experimental procedures}

\section{Plant material and plant growth conditions}

The wild-type maize plants used throughout this study were $F_{1}$ hybrids from a cross between W23 and stock 924A from the Maize Genetics Cooperation Stock Center (http://maizecoop.cropsci.uiuc. edu). The 924A stock carries white deficiency, and is of a genetic background that is one quarter M14/W23 and three quarters undefined. This 5/8 W23 hybrid shows superior performance in shoot apex culture, compared with the W23 inbred line (El, unpublished data), which was required for these experiments. In this line, L1-L4 differentiated entirely as juvenile leaves, whereas $L 8$ and above differentiated entirely as adult leaves. The L5-L7 leaves were transition leaves, showing juvenile traits at the first-differentiating tip, and adult traits closer to the base of the leaf blade. Maize kernels were sown in Jiffy Plus potting mix (Jiffy Products of America Inc., http:// www.jiffypot.com) in $8.5-\mathrm{cm}$-diameter plastic pots. Plants were grown in the greenhouse with a 16-h light/8-h dark photoperiod provided by $1000 \mathrm{~W}$ high-pressure sodium and metal halide lamps.

\section{Shoot apex culture}

Shoot apices used for shoot apex culture were harvested from W23/ 924A hybrid plants at approximately 3 weeks after planting, when they had initiated 12 leaves in total and showed five or six expanded leaves. Shoot apices consisting of the meristem plus the two newest leaf primordia were cultured. Rejuvenated L3 and L4 leaves thus correspond to the first two leaves formed by the meristem after its excision from the plant, and would have been L13 and L14 on the plant had the shoot apex not been excised. Shoot apex culture was performed as described previously (Irish and Nelson, 1988).

\section{RNA isolation}

Leaf primordia at P6 (i.e. the stage that a leaf reaches during the period in which five additional leaves have been initiated by the shoot meristem, which corresponds to a leaf length between 4 and $6 \mathrm{~mm}$ in the line used here) were collected for L4 and L9 from seed-derived plants, and L3 or L4 from culture-derived plants. Total RNA was extracted from samples consisting of approximately 24 leaf primordia using Trizol reagent (Invitrogen, http:// www.invitrogen.com) following the manufacturer's recommendations.

\section{Experimental design and microarray procedures}

For each of six biological replications, each of the three pairwise comparisons of P6-staged leaf primordia from L4, L9 and RL3/4 was made on one slide. With six biological replications and three slides per replication ( $L 4$ versus $L 9$, $L 9$ versus $R L 3 / 4$ and $R L 3 / 4$ versus $L 4$ ), this replicated loop design used a total of 18 slides. To ensure dye balance, each of the 18 target samples was measured once with $\mathrm{Cy} 3$ labeling, and once with Cy5 labeling.

Fluorescently labeled CDNA targets were prepared and hybridized to a 12 160-element cDNA microarray chip (Generation II, version B) according to the protocol available at http://schnablelab. plantgenomics.iastate.edu/resources/protocols. The microarray was generated at the Center for Plant Genomics at lowa State University (http://www.plantgenomics.iastate.edu/maizechip; Nakazono et al., 2003).

\section{Data acquisition}

Microarray slides were scanned with a ScanArray 5000 (Hewlett Packard Inc., http://www.hp.com). A multiple scanning method similar to that described by Skibbe et al. (2006) was implemented. Fluorescent signal intensities were determined using IMAGENE 5.0 (Biodiscovery, http://www.biodiscovery.com). Scan pairs with Cy3 and $\mathrm{Cy} 5$ scan intensities of similar median values over all replicate slides constituted a scan set. Two scan sets (a low- and high-scan set) that had approximate median natural log signals of 6 and 7 prior to normalization were selected from all six scans of the 18 slides for analysis.

\section{Data normalization}

The Lowess normalization method (Dudoit et al., 2002) was applied to the log of the background-corrected raw signal intensity (signal intensity minus the median background intensity) to remove signal-intensity-dependent dye effects on each slide. Lowess normalization was performed separately for each slide to avoid the introduction of dependence among the independent biological replicates. The normalized data for each slide/dye combination were mean-centered (each individual value associated with a particular slide/dye combination minus the average value associated with the particular slide/dye combination), so that expression measures would be comparable across all slides. As a result, negative (positive) values were indicative of a particular transcript being expressed below (above) the average for a particular slide/dye combination. 


\section{Data analysis}

Of the 12160 spots contained on the Generation-II version-B cDNA array, 1520 'empty' or 'bad-PCR' spots were removed from the data set prior statistical analysis. A mixed linear model analysis (Wolfinger et al., 2001) of the normalized log-scale signal intensities for each of the 10640 spots on the array was performed to identify significant transcripts differentially represented among juvenile, adult and culture-rejuvenated leaves. The mixed linear model included fixed effects for leaf number/type (L4, L9 or RL3/4) and dye (Cy3 or Cy5), as well as random effects for day of hybridization, replication, slide and sample. As part of each mixed linear model analysis, a Student's $t$-test for differential gene expression was conducted for each pair-wise comparison of leaf number/type. The proportion of false-positive results among all genes with $P \leq 0.005$ was estimated by applying the method of Fernando et al. (2004). In addition to $P$-value analysis, 'fold-change' estimates and $95 \%$ confidence intervals associated with the fold-change estimates were computed as part of the mixed linear model analysis.

An additional 2754 spots were removed from the data set after data analysis because of concerns regarding the quality of the associated DNA sequences. Data (GEO GSE9430) reported in this study were derived from the remaining 7886 'informative' spots.

\section{Semi-quantitative RT-PCR}

Results from the microarray experiment were validated using an independent replicate of total RNA that was isolated from P6-stage primordia for L4 and L9. First-strand cDNA was synthesized using $1 \mu \mathrm{g}$ of total RNA, $25 \mu \mathrm{g} \mathrm{ml}^{-1}$ oligo(dT) 22 primer, $0.5 \mathrm{~mm}$ deoxyribonucleotide triphosphate (dNTP) mix, 1x first-strand buffer, $0.01 \mathrm{M}$ DTT, $40 \mathrm{U}$ of RNaseOUT (Invitrogen) and $200 \mathrm{U}$ of Superscript II reverse transcriptase (Invitrogen) following the manufacturer's directions. One-twentieth of the reaction volume was used as the template for amplifications. The number of PCR cycles used for all genes is indicated in Figure 5. Primers used for amplifications are listed in Table S2.

\section{RNA blot analysis}

Total RNA $(15 \mu \mathrm{g})$ per leaf was loaded in each lane of a $1 \%(\mathrm{w} / \mathrm{v})$ agarose formaldehyde denaturing gel, and was blotted onto Hybond-N membranes (Amersham Biosciences, http://www.amersham.com). Filters were hybridized with DNA probes corresponding to the EST clone on the array. EST-specific clones were generated by PCR amplification of the cDNA insert using gene-specific primers (IDT, http://eu.idtdna.com; Table S2) in reactions with 1× PCR buffer, $0.2 \mu \mathrm{m}$ of each primer, $0.2 \mathrm{~mm}$ dNTPs and $0.04 \mathrm{U}$ of Taq polymerase (NEB, http://www.neb.com). Thirty-five cycles were used to amplify all ESTs. Amplified DNA products were purified using a OlAquick Gel Extraction kit (Qiagen, http://www.qiagen.com). DNA probes were labeled with $\left[\alpha^{-32} \mathrm{P}\right]$ deoxycytidine triphosphate (dCTP) using a random prime method. The hybridization of probes and subsequent washings were performed as described by Church and Gilbert (1984), with slight modifications.

\section{Acknowledgements}

We thank Peter Quail and Sarah Hake for the Ubiquitin clones. Assistance from other members of the Schnable lab, especially Debbie Chen, Hailing Jin, Dave Skibbe, Eddy Yeh and Pengcheng $\mathrm{Lu}$, is gratefully acknowledged. We also thank two anonymous reviewers for their valuable suggestions. The Avis Cone Summer Research Fellowship provided partial support for JS.

\section{Supporting Information}

Additional supporting information may be found in the online version of this article:

Figure S1. Expressed sequence tags (ESTs) that were differentially expressed between juvenile leaf 4 , adult leaf 9 and culture-rejuvenated leaves $3 / 4$ in the high-intensity scan data set.

Table S1. Juvenile-induced expressed sequence tags (ESTs). These ESTs show at least a twofold increase in expression at $P \leq 0.005$ in juvenile tissues compared with adult tissues.

Table S2. Primers for the PCR amplification of selected phaseinduced expressed sequence tags (ESTs).

Please note: Wiley-Blackwell are not responsible for the content or functionality of any supporting materials supplied by the authors. Any queries (other than missing material) should be directed to the corresponding author for the article.

\section{References}

Abedon, B.G., Hatfield, R.D. and Tracy, W.F. (2006) Cell wall composition in juvenile and adult leaves of maize (Zea mays L.). J. Agric. Food. Chem. 54, 3896-3900.

Aukerman, M.J. and Sakai, H. (2003) Regulation of flowering time and floral organ identity by a microRNA and its APETALA2-like target genes. Plant Cell, 15, 2730-2741.

Berardini, T.Z., Bollman, K., Sun, H. and Poethig, R.S. (2001) Regulation of vegetative phase change in Arabidopsis thaliana by cyclophilin 40. Science, 291, 2405-2407.

Bongard-Pierce, D.K., Evans, M.M.S. and Poethig, R.S. (1996) Heteroblastic features of leaf anatomy in maize and their genetic regulation. Int. J. Plant Sci. 157, 331-340.

Brink, R.A. (1962) Phase change in higher plants and somatic cell heredity. Q. Rev. Biol. 37, 1-22.

Brusslan, J.A. and Tobin, E.M. (1992) Light-independent developmental regulation of cab gene expression in Arabidopsis thaliana seedlings. Proc. Natl Acad. Sci. USA, 89, 7791-7795.

Buckner, B., Beck, J., Browning, K. et al. (2007) Involving undergraduates in the annotation and analysis of global gene expression studies: creation of a maize shoot apical meristem expression database. Genetics, 176, 741-747.

Chang, Y.C. and Walling, L.L. (1992) Chlorophyll a/b-binding protein genes are differentially expressed during soybean development. Plant Mol. Biol. 19, 217-230.

Chory, J., Nagpal, P. and Peto, C.A. (1991) Phenotypic and genetic analysis of det2, a new mutant that affects light-regulated seedling development in Arabidopsis. Plant Cell, 3, 445-459.

Christensen, A.H. and Quail, P.H. (1989) Sequence analysis and transcript regulation by heat shock of polyubiquitin transcripts from maize. Plant Mol. Biol. 12, 619-632.

Chuck, G., Cigan, A.M., Saeteurn, K. and Hake, S. (2007) The heterochronic maize mutant Corngrass 1 results from overexpression of a tandem microRNA. Nat. Genet. 39, 544-549.

Church, G.M. and Gilbert, W. (1984) Genomic sequencing. Proc. Natl Acad. Sci. USA, 81, 1991-1995.

Dudoit, S., Yang, Y.H., Callow, M.J. and Speed, T.P. (2002) Statistical methods for identifying differentially expressed genes in replicated cDNA microarray experiments. Stat. Sin. 12, 111-139.

Evans, M.M.S. and Poethig, R.S. (1995) Gibberellins promote vegetative phase change and reproductive maturity in maize. Plant Physiol. 108, 475-487.

Evans, M.M.S. and Poethig, R.S. (1997) The viviparous8 mutation delays vegetative phase change and accelerates the rate of seedling growth in maize. Plant J. 12, 769-779. 
Evans, M.M.S., Passas, H. and Poethig, R.S. (1994) Heterochronic effects of glossy 15 mutations on epidermal cell identity in maize. Development, 120, 1971-1981.

Fernando, R.L., Nettleton, D., Southey, B.R., Dekkers, J.C.M., Rothschild, M.F. and Soller, M. (2004) Controlling the proportion of false positives in multiple dependent tests. Genetics, 166, 611619.

Freeling, M. (1992) A conceptual framework for maize leaf development. Dev. Biol. 153, 44-58.

Freeling, M. and Lane, B. (1994) The maize leaf. In The Maize Handbook (Freeling, M. and Walbot, V., eds). New York: Springer Verlag, pp. 17-28.

Gandikota, M., Birkenbihl, R.P., Hohmann, S., Cardon, G.H., Saedler, H. and Huijser, P. (2007) The miRNA 156/157 recognition element in the $3^{\prime}$ UTR of the Arabidopsis SBP box gene SPL3 prevents early flowering by translational inhibition in seedlings. Plant J. 49, 683-693.

Hackett, W.P. (1985) Juvenility, maturation and rejuvenation in woody plants. Hortic. Rev. 7, 109-155.

He, Z.H., Li, J., Sundqvist, C. and Timko, M.P. (1994) Leaf developmental age controls expression of genes encoding enzymes of chlorophyll and heme biosynthesis in pea (Pisum sativum L.) Plant Physiol. 106, 537-546.

Irish, E.E. and Karlen, S. (1998) Restoration of juvenility in maize shoots by meristem culture. Int. J. Plant Sci. 159, 695-701.

Irish, E.E. and McMurray, D. (2006) Rejuvenation by shoot apex culture recapitulates the developmental increase of methylation at the maize gene Pl-Blotched. Plant Mol. Biol. 60, 747758

Irish, E.E. and Nelson, T. (1988) Development of maize plants from cultured shoot apices. Planta, 175, 9-12.

Kerstetter, R.A. and Poethig, R.S. (1998) The specification of leaf identity during shoot development. Annu. Rev. Cell Dev. Biol. 14, 373-398.

Langdale, J.A., Rothermel, B.A. and Nelson, T. (1988) Cellular patterns of photosynthetic gene expression in developing maize leaves. Genes Dev. 2, 106-115.

Lauter, N., Kampani, A., Carlson, S., Goebel, M. and Moose, S.P. (2005) microRNA172 down-regulates glossy15 to promote vegetative phase change in maize. Proc. Natl Acad. Sci. USA, 102, 9412-9417.

Millar, A.A., Clemens, S., Zachgo, S., Giblin, E.M., Taylor, D.C. and Kunst, L. (1999) CUT1, an Arabidopsis gene required for cuticular wax biosynthesis and pollen fertility, encodes a very-long-chain fatty acid condensing enzyme. Plant Cell, 11, 825-838.

Moose, S.P. and Sisco, P.H. (1994) Glossy15 controls the epidermal juvenile-to-adult phase transition in maize. Plant Cell, 6, 13431355.

Moose, S.P. and Sisco, P.H. (1996) Glossy15, an APETALA2-like gene from maize that regulates leaf epidermal cell identity. Genes Dev. 10, 3018-3027.

Moreno, M.A., Harper, L.C., Krueger, R.W., Dellaporta, S.L. and Freeling, M. (1997) liguleless 1 encodes a nuclear-localized protein required for induction of ligules and auricles during maize leaf organogenesis. Genes Dev. 11, 616-628.

Nakazono, M., Qiu, F., Borsuk, L.A. and Schnable, P.S. (2003) Lasercapture microdissection, a tool for the global analysis of gene expression in specific plant cell types: identification of genes expressed differentially in epidermal cells or vascular tissues of maize. Plant Cell, 15, 583-596.
Orkwiszewski, J. and Poethig, R.S. (2000) Phase identity of the maize leaf is determined after leaf initiation. Proc. Natl Acad. Sci. USA, 97, 10631-10636.

Pighin, J.A., Zheng, H., Balakshin, L.J., Goodman, I.P., Western, T.L., Jetter, R., Kunst, L. and Samuels, A.L. (2004) Plant cuticular lipid export requires an ABC transporter. Science, 306, 702-704.

Poethig, R.S. (1988) Heterochronic mutations affecting shoot development in maize. Genetics, 119, 959-973.

Poethig, R.S. (1990) Phase change and the regulation of shoot morphogenesis in plants. Science, 250, 923-930.

Pratt, W.B., Krishna, P. and Olsen, L.J. (2001) Hsp90-binding immunophilins in plants: protein movers. Trends Plant Sci. 6, 5458.

Ramsperger, V.C., Summers, R.G. and Berry, J.O. (1996) Photosynthetic gene expression in meristems and during initial leaf development in a $\mathrm{C}_{4}$ dicotyledonous plant. Plant Physiol. 111, 999-1010.

Reimer, U. and Fischer, G. (2002) Local structural changes caused by peptidyl prolyl cis/trans isomerization in the native state of proteins. Biophys. Chem. 96, 203-212.

Rhoades, M.W., Reinhart, B.J., Lim, L.P., Burge, C.B., Bartel, B. and Bartel, D.P. (2002) Prediction of plant microRNA targets. Cell, 110, 513-520.

Romano, P., Gray, J., Horton, P. and Luan, S. (2005) Plant immunophilins: functional versatility beyond protein maturation. New Phytol. 166, 753-769.

Schwab, R., Palatnik, J.F., Riester, M., Schommer, C., Schmid, M. and Weigel, D. (2005) Specific effects of microRNAs on the plant transcriptome. Dev. Cell, 8, 517-527.

Simpson, G.G., Gendall, A.R. and Dean, C. (1999) When to switch to flowering. Annu. Rev. Cell Dev. Biol. 15, 519-550.

Skibbe, D.S., Wang, X., Zhao, X., Borsuk, L.A., Nettleton, D. and Schnable, P.S. (2006) Scanning microarrays at multiple intensities enhances discovery of differentially expressed genes. Bioinformatics, 22, 1863-1870.

Spencer, M.W.B., Casson, S.A. and Lindsey, K. (2007) Transcriptional profiling of the Arabidopsis embryo. Plant Physiol. 143 924-940.

Steeves, T.A. and Sussex, I.M. (1988) Patterns of Plant Development, 2nd edn. New York: Cambridge University Press.

Vega, S.H., Sauer, M., Orkwiszewski, J.A.J. and Poethig, R.S. (2002) The early phase change gene in maize. Plant Cell, 14, 133-147.

Wang, H., Nussbaum-Wagler, T., Li, B., Zhao, Q., Vigouroux, Y., Faller, M., Bomblies, K., Lukens, L. and Doebley, J.F. (2005) The origin of the naked grains of maize. Nature, 436, 714-719.

Wolfinger, R.D., Gibson, G., Wolfinger, E.D., Bennett, L., Hamadeth L., Bushel, P., Afshari, C. and Paules, R.S. (2001) Assessing gene significance from cDNA microarray expression data via mixed models. J. Comput. Biol. 8, 625-637.

Woo, H.-H., Hackett, W.P. and Das, A. (1994) Differential expression of a chlorophyll $\mathrm{a} / \mathrm{b}$ binding protein gene and a proline rich gene in juvenile and mature phase English ivy (Hedera helix). Physiol. Plant, 92, 69-78.

Wu, G. and Poethig, R.S. (2006) Temporal regulation of shoot development in Arabidopsis thaliana by miR165 and its target SPL3. Development, 133, 3539-3547.

Xie, K., Wu, C. and Xiong, L. (2006) Genomic organization, differential expression, and interaction of SOUAMOSA promoterbinding-like transcription factors and microRNA156 in rice. Plant Physiol. 142, 280-293. 